\title{
Study on hexane adsorption in zeolite ITQ-29 by molecular simulation
}

\author{
Miguel A. Granato • Thijs J.H. Vlugt • \\ Alírio E. Rodrigues
}

Received: 1 February 2008 / Revised: 6 July 2008 / Accepted: 22 July 2008 / Published online: 12 September 2008

(c) Springer Science+Business Media, LLC 2008

\begin{abstract}
Adsorption isotherms and isosteric heat of adsorption of $n$-hexane in zeolite ITQ-29 were simulated using the Configurational Bias Monte Carlo (CBMC) technique in the grand-canonical $(\mu V T)$ ensemble and compared with experimental results published by Gribov et al. and obtained by IR spectroscopy where the fractional loadings of $n$-hexane in ITQ-29 are presented in units from integral intensities of the absorption bands [u.a.]. In this work we present the simulation loadings of $n$-hexane in ITQ-29 converted to fractional coverages and compared to the experimental results. The simulations were performed using a united atom force field. In addition, we calculated equilibrium adsorption isotherms of ethane and propane in ITQ-29 in excellent agreement with published experiments. This force field successfully reproduces adsorption properties of linear alkanes in cation-free LTA zeolite and is suitable for fast and accurate adsorption data predictions.
\end{abstract}

Keywords Molecular simulation - Adsorption - Monte Carlo $\cdot$ Zeolite ITQ-29 $\cdot$ Hexane

M.A. Granato · A.E. Rodrigues $(\bowtie)$

Laboratory of Separation and Reaction Engineering (LSRE), Department of Chemical Engineering, Faculty of Engineering, University of Porto, Rua Dr. Roberto Frias, s/n, 4200-465, Porto, Portugal

e-mail: arodrig@fe.up.pt

T.J.H. Vlugt

Process and Energy Laboratory, Delft University of Technology, Leeghwaterstraat 44, 2628 CA, Delft, The Netherlands

\section{Introduction}

The use of zeolites in catalytic and separation processes became of fundamental importance due to the savings in energy consumption and yield efficiency. Hundreds of different types of zeolites have been synthesized and tested in a wide range of processes (Breck 1984; Corma 2003).

Recent studies on $n$-hexane adsorption in zeolite ITQ-29 among others were reported by Gribov et al. (2005). Equilibrium isotherms and heats of adsorption were determined experimentally by IR spectroscopy. The loadings of hexane in ITQ-29 are presented in arbitrary units from integral intensities of the absorption bands [u.a.]. Zeolite ITQ-29 is the cation-free type of LTA framework, recently synthesized (Corma et al. 2004).

The heats of adsorption of $n$-alkanes for various all-silica zeolites are well documented, either experimentally or by molecular simulation (Dubbeldam 2005; Bates et al. 1996; Gribov et al. 2005) and pulsed field gradient (PFG) nuclear magnetic resonance (NMR) spectroscopy was used to measure self-diffusion coefficients of propylene in ITQ-29 (Hedin et al. 2007). However, molecular simulation studies on adsorption isotherms of $n$-hexane in cation-free LTA are not found in literature, although Molecular Dynamics (MD) studies on diffusion of $n$-alkanes are reported (Dubbeldam 2005; Dubbeldam et al. 2005; Schüring et al. 2002).

The objective of this work is to apply the CBMC molecular simulation technique to calculate adsorption properties of $n$-hexane in zeolite ITQ-29 and to compare them with those obtained by classical calculation methods based on published experimental data. 


\section{Computational model}

\subsection{Zeolite ITQ-29}

The cation-free LTA-type structure (The Atlas of Zeolite Structure Types 2007) has a cubic space group $F m-3 c$ with $\mathrm{a}=24.6 \AA$. The crystallographic unit cell has two interconnecting, 3-dimensional channel systems, one consisting of 8 large spherical cages (named $\alpha$-cages) of approximately $11.4 \AA$ interconnected via windows of about $4.2 \AA$ diameter and the other consisting of $\beta$-cages with average diameter of $6.6 \AA$ alternating with the $\alpha$-cages and separated by $2.2 \AA$ openings. An $n$-hexane molecule does fit in the $\beta$-cages, but it is not accessible from the main $\alpha$-cages through the small $2.2 \AA$ apertures. However, the CBMC method randomly inserts a guest molecule atom by atom inside the zeolite so molecules could actually enter these cages. To prevent false higher loadings, the $\beta$-cages are artificially blocked according to the procedure outlined in Dubbeldam et al. (2005). The zeolite framework is assumed to be rigid as the influence of zeolite framework flexibility is small in adsorption (Vlugt 2002). In this work, a simulation box which represents the LTA framework of composition $\mathrm{Si}_{192} \mathrm{O}_{384}$ was used and periodic boundary conditions were applied.

\subsection{Simulation technique}

Adsorption isotherms were calculated by using the configurational-bias Monte Carlo (CBMC) technique in the grand-canonical $(\mu V T)$ ensemble. In MC simulations, the zeolite crystals are allowed to exchange molecules with a reservoir of molecules at a fixed chemical potential. The simulations are performed in cycles and in each cycle one move is chosen at random with a fixed probability of four types of moves: molecule translation $(0.15)$, rotation around the center of mass $(0.15)$, exchange with the reservoir $(0.55)$ and partial regrowth of a molecule (0.15). The number of cycles was $2 \times 10^{6}$. For a more detailed description of this simulation technique, the reader is referred to Frenkel and Smit (2002) and Vlugt et al. (1999).

\subsection{Force field}

The $n$-hexane molecule is described by a united-atom model, in which the $\mathrm{CH}_{n}$ groups are considered as a single interaction center without charge. In the CBMC algorithm, the chain is built from a first united atom, placed at a random position. The second atom is added to the first one and a harmonic bonding potential is used for the bond length. The chain is grown segment by segment. The bond bending between three neighboring beads is modeled by a harmonic cosine bending potential and the torsional angle is controlled by a Ryckaert-Bellemans potential. The bond length is fixed (1.53 $\AA$ ). The carbon-carbon and carbonzeolite interactions are described by a 12-6 Lennard-Jones potential. The interactions between different pseudo-atoms are determined by the Lorentz-Berthelot mixing rules.

$\sigma_{i j}=\frac{1}{2}\left(\sigma_{i i}+\sigma_{j j}\right), \quad \varepsilon_{i j}=\left(\varepsilon_{i i} \cdot \varepsilon_{j j}\right)^{1 / 2}$

The force field parameters used in this work are shown in Tables 1 and 2. Details on the force field are found in Calero et al. (2004) and Martin and Siepmann (1998).
Table 1 Intramolecular Force Field Parameters (Calero et al. 2004)

Table 2 Parameters for United Atom (UA) Force Field (Calero et al. 2004)

\begin{tabular}{lllll}
\hline & & $\mathrm{O}$ & $\mathrm{CH}_{3}$ & $\mathrm{CH}_{2}$ \\
\hline $\mathrm{CH}_{3}$ & $\varepsilon / k_{\mathrm{B}}(\mathrm{K})$ & 93.00 & 108.00 & 77.70 \\
& $\sigma(\AA)$ & 3.48 & 3.76 & 3.86 \\
$\mathrm{CH}_{2}$ & $\varepsilon / k_{\mathrm{B}}(\mathrm{K})$ & 60.50 & 77.70 & 56.00 \\
& $\sigma(\AA)$ & 3.58 & 3.86 & 3.96 \\
\hline
\end{tabular}

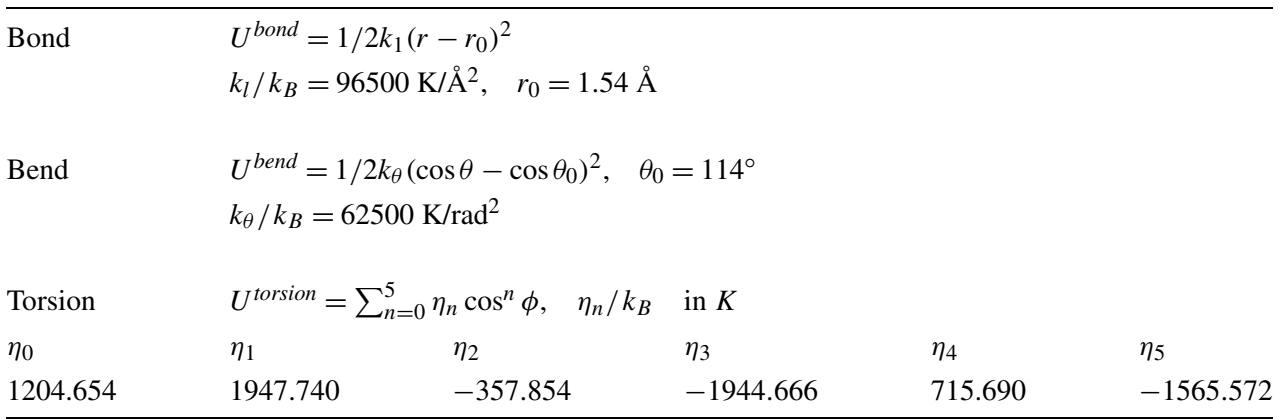




\section{Results}

\subsection{Adsorption of $n$-hexane in zeolite ITQ-29}

From the Langmuir approach the fractional coverage of $n$-hexane in the adsorbed phase $\theta_{i}$ can be calculated:

$\theta_{i}=\frac{q_{i}}{q_{\max }}=\frac{A_{i}}{A_{\max }}=\frac{K_{S} P}{1+K_{S} P}$

where $q_{i}=$ adsorbed phase concentration of $n$-hexane; $q_{\max }=$ maximum adsorbed phase concentration; $A_{i}=$ integral intensity of the given IR band; $A_{\max }=$ integral intensity of the same band at maximum loading; $K_{S}=$ sorption constant; $P=$ pressure.

Thus, simulation loadings of $n$-hexane in ITQ-29 can be converted to fractional coverages and compared to the experimental fractional coverages. To do so, it is necessary to determine the actual maximum integral intensities and, then, to calculate the correct fractional coverage for the experiments. The isotherm was plotted for a low pressure range and fitted by the Langmuir equation.

The values of the sorption constant, $K_{S}$ were directly taken from the experimental data of Gribov et al. (2005). From such information one can apply the method described in Breck (1984), as follows:

Rewriting (2):

$$
\frac{P}{A}=\frac{1}{A_{\max } K_{S}}+\frac{P}{A_{\max }}
$$

The value of $P / A$ plotted against $P$ gives a straight line with a slope of $1 / A_{\max }$. Table 3 shows the calculations results: the differences between the values of $A_{\max }$ at different temperatures are not significant.

\subsection{Heats of adsorption}

The Arrhenius equation:

$$
K_{S}(T)=C \exp ^{(-\Delta H / R T)}
$$

in the form:

$\ln K_{S}(T)=\ln C+\frac{(-\Delta H)}{R} \frac{1}{T}$

Table 3 Determination of $A_{\max }$

\begin{tabular}{ll}
\hline Temp. $(\mathrm{K})$ & $A_{\max }($ u.a. $)=1 / \mathrm{a}$ \\
\hline 374 & 45.331 \\
415 & 43.592 \\
434 & 45.039 \\
453 & 42.824 \\
\hline
\end{tabular}

generates a plot of $\ln K_{S}(T)$ versus $1 / T$ which slope is $(-\Delta H / R)$ and the $y$-intercept is $\ln C$. The heat of sorption $(-\Delta H)$ at a given temperature $T$. Note: $C$ is a pre-exponential factor. From the adsorption isotherms obtained by GCMC simulations, the isosteric heat of adsorption was calculated by the Clausius-Clapeyron relationship $(39.7 \mathrm{~kJ} / \mathrm{mol})$, for a loading of 12 molecules per unit cell, in excellent agreement with the value of $39 \mathrm{~kJ} / \mathrm{mol}$ reported by Gribov et al. (2005). The isosteric heat of adsorption in low coverage regime ( 2 molecules/unit cell), obtained by the Clausius-Clapeyron relationship was found to be $34.6 \mathrm{~kJ} / \mathrm{mol}$.

Additionally, heats of adsorption of $n$-hexane in zeolite ITQ-29 have been obtained by GCMC ensemble from combined energy/particle fluctuations (Karavias and Myers 1991). For the simulations the total number of cycles was at least $2 \times 10^{6}$. The calculated heats of adsorption as a function of the loading are shown in Fig. 1. The heat of sorption of $n$-hexane in ITQ-29 was found to be $50.5 \mathrm{~kJ} / \mathrm{mol}$ for a loading of 20 molecules per unit cell, which is in excellent agreement with the value of $52.3 \mathrm{~kJ} / \mathrm{mol}$ for a similar loading of 24 molecules per unit cell, corresponding to 3 molecules/supercage, reported by Gribov et al. (2005). It confirms that theoretically calculated heats of sorption are higher than those obtained from experimental data. However, for a loading range between 8 and 16 molecules per unit cell ( 1 to 2 molecules per supercage), the sorption energy is not too much dependent of the loading and the values remain among 36 and $40 \mathrm{~kJ} / \mathrm{mol}$. Table 4 shows results of enthalpy of adsorption from literature, compared with our calculations.

\section{Hexane/ITQ-29 isotherms by molecular simulation}

The experimental study was carried out for a very low pressure range. To compare the experimental fractional loadings with the simulations it is necessary to determine the maximum loading $\left(A_{\max }\right)$ which, as previously described, was calculated by linearization of $P / A$ plotted against $P$, the slope of the resulting straight line being $1 / A_{\max }$.

The maximum capacity was found to be 20 molecules per unit cell by molecular simulation. Force field parameters from Calero et al. (2004) have been used. The estimated maximum capacity in units of integral intensities of the absorption bands [u.a.] were also calculated by using (1), for the same range of pressures. The results can be compared in fractional coverages and are presented in Fig. 2. The fractional coverages for low-pressure range are shown in Fig. 3. In order to validate our results, ethane and propane isotherms have been calculated by CBMC simulations and compared with experimental data reported by Hedin et al. (2007). The agreement between experiments and simulations is very good, as is shown in Fig. 4. 
Fig. 1 Heats of adsorption $n$-hexane in ITQ-29 as a function of the loading (a)

Heats of adsorption of $n$-hexane in zeolite ITQ-29

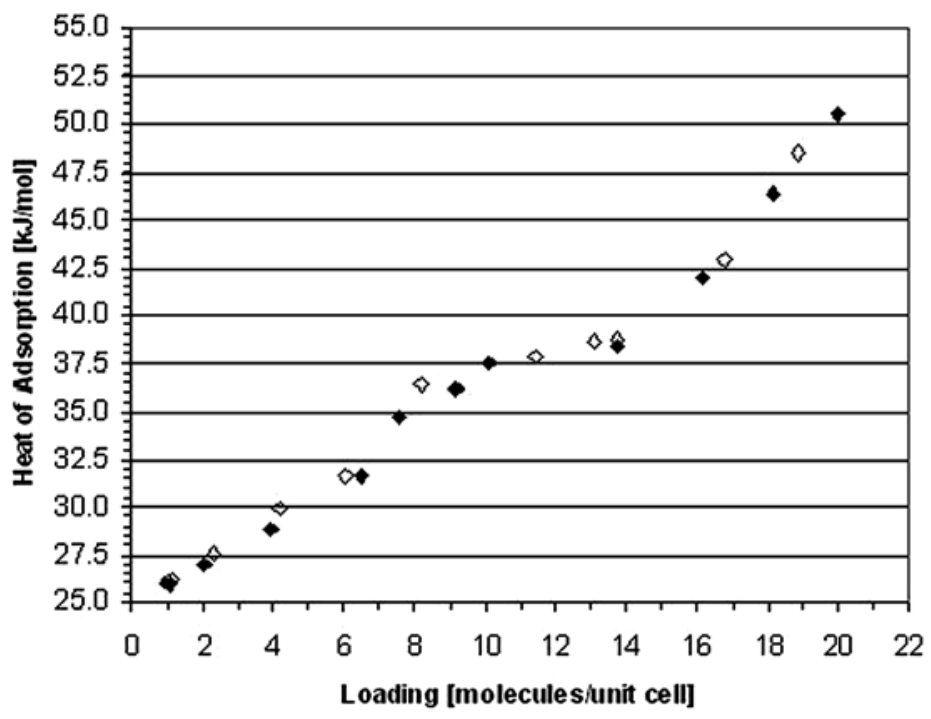

This work

CB-GCMC Simulations

- $374 \mathrm{~K}$

$\diamond 453 \mathrm{~K}$ (b)

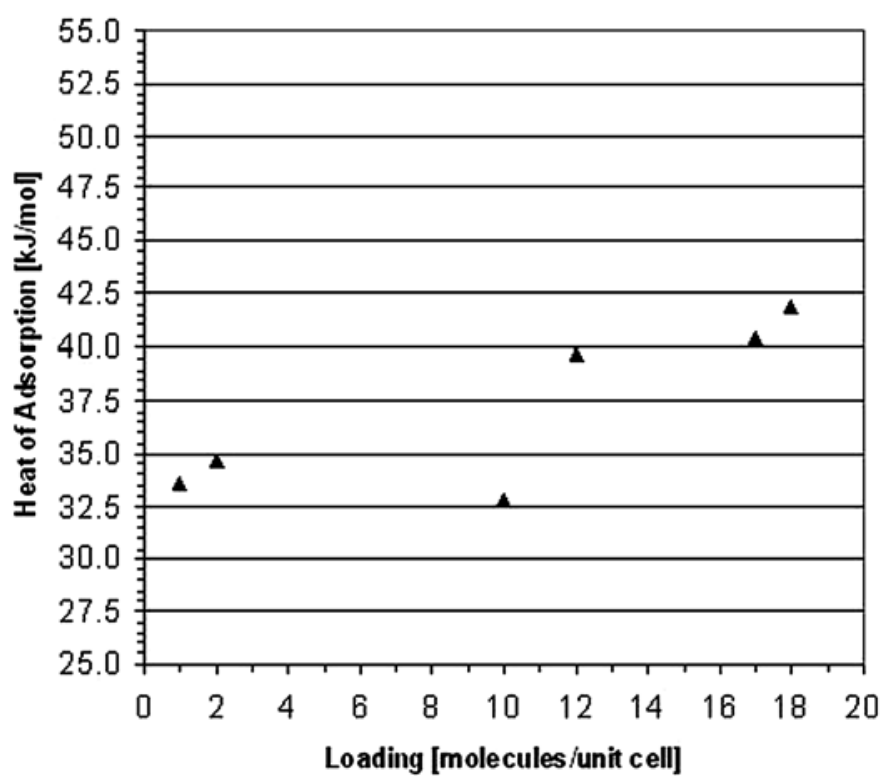

^ Clausius-Clapeyron from molecular simulations isothems

Table 4 Heats of $n$-hexane adsorption

\begin{tabular}{|c|c|c|}
\hline Reference & $(\mathrm{kJ} / \mathrm{mol})$ & Method \\
\hline Gribov et al. (2005) & $\begin{array}{l}39.0 \\
52.3 \\
54.1 \\
46.6\end{array}$ & $\begin{array}{l}\text { From IR } \\
\text { From calculations }{ }^{\text {a }}(24 \text { molecules/unit cell }) \\
16 \text { molecules/unit cell } \\
8 \text { molecules/unit cell }\end{array}$ \\
\hline Bates et al. (1996) & 41.2 & \\
\hline This work ${ }^{\mathrm{b}}$ & $\begin{array}{l}34.7 \\
39.7 \\
50.51 \\
27.7\end{array}$ & $\begin{array}{l}\text { Clausius-Clapeyron relationship ( } 2 \text { molecules/unit cell) } \\
\text { Clausius-Clapeyron relationship ( } 12 \text { molecules/unit cell) } \\
\text { Molecular simulation ( } 20 \text { molecules/unit cell) } \\
\text { Molecular simulation ( } 2 \text { molecules/unit cell) }\end{array}$ \\
\hline
\end{tabular}

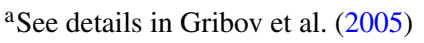

${ }^{\mathrm{b}}$ Other values shown in Fig. 1a and b 
Fig. 2 Fractional coverages of $n$-hexane in ITQ-29

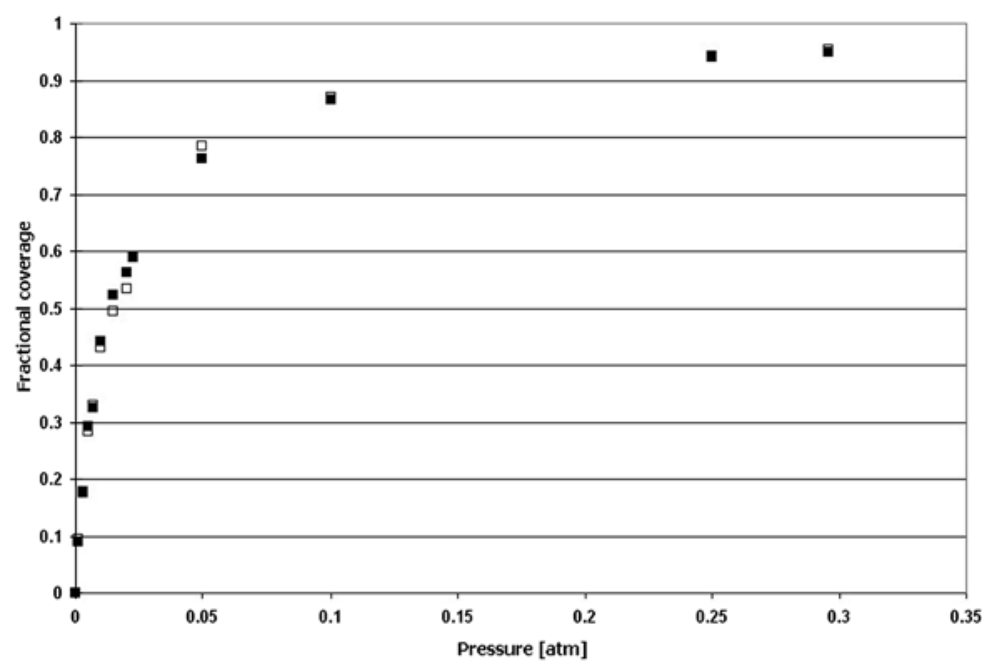

- Gribov et al. (2005) 口 Simulations - this work

$374 \mathrm{~K}$

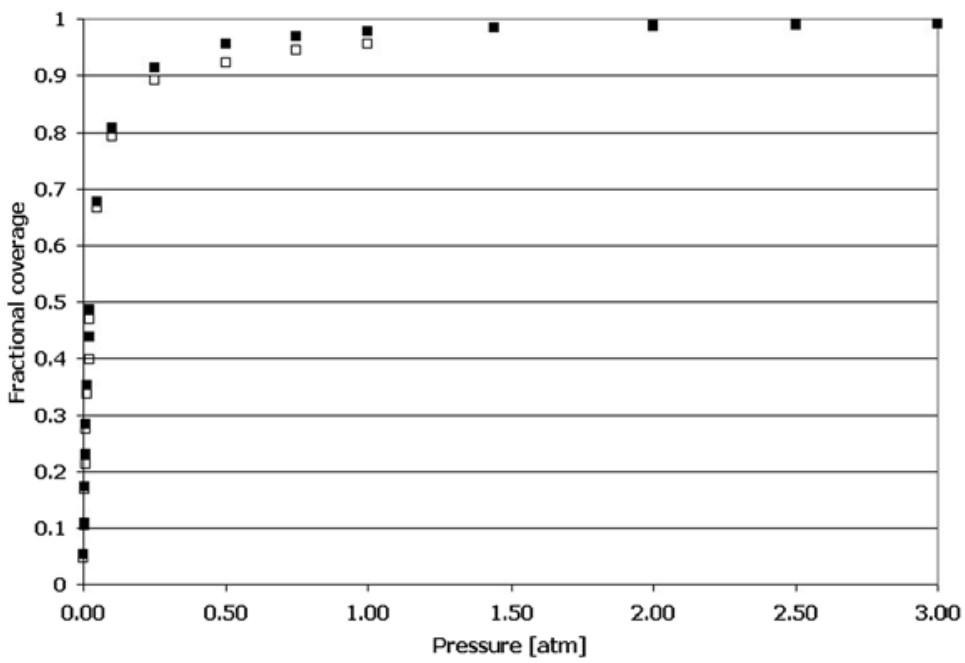

- Gribov et al. (2005) 口 Simulations - this work $415 \mathrm{~K}$

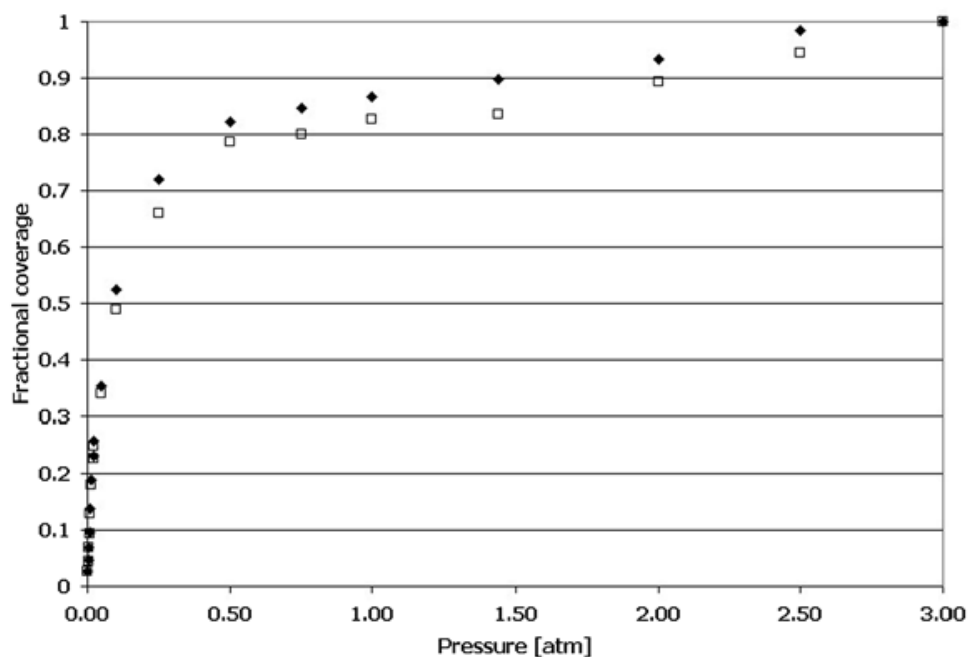

- Gribov et al. (2005)

口Simulations - this work $453 \mathrm{~K}$

A snapshot of the equilibrium configuration of 20 adsorbed $n$-hexane molecules in the large $\alpha$-cages of zeolite ITQ-29 is shown in Fig. 5. At this high loading, the mole- cules are arranged in different ways to best fill the cavities. Shadowed parts of the snapshot represent a 3-dimensional depth cueing from the frontal plane of view. 
Fig. 3 Fractional coverages of $n$-hexane in ITQ-29—range of low pressures

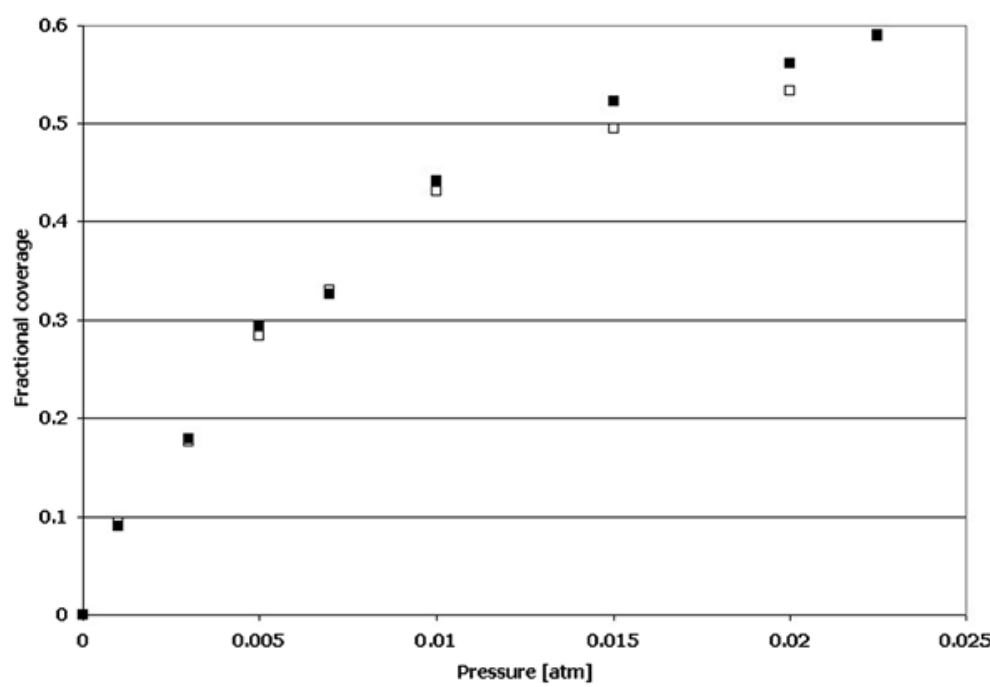

- Gribov et al. (2005) 口Simulations - this work $374 \mathrm{~K}$

- Gribov et al. (2005) 口Simulations - this work $415 \mathrm{~K}$
- Gribov et al. (2005)

口 Simulations - this work $453 K$ 


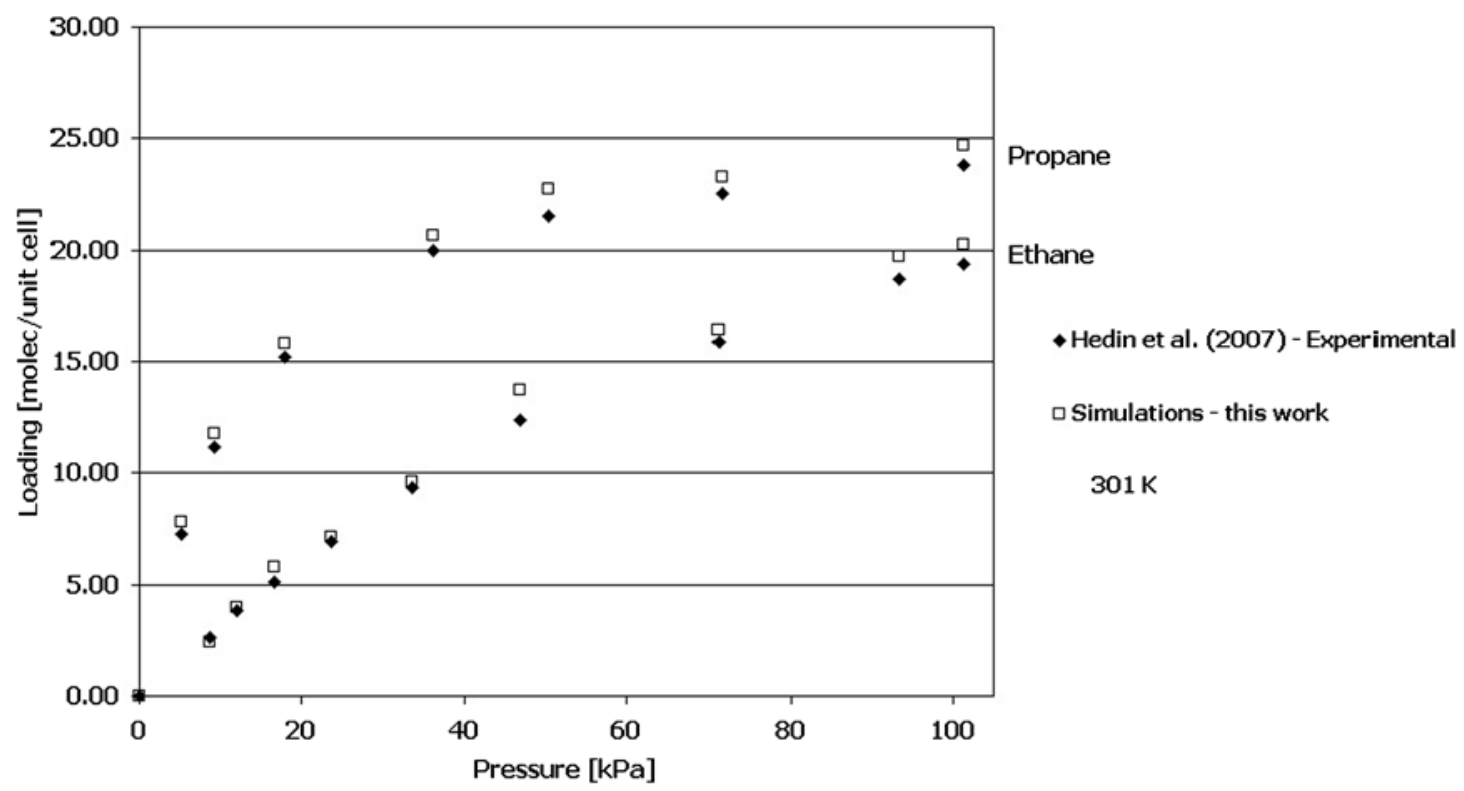

Fig. 4 Equilibrium adsorption isotherms of ethane and propane in ITQ-29

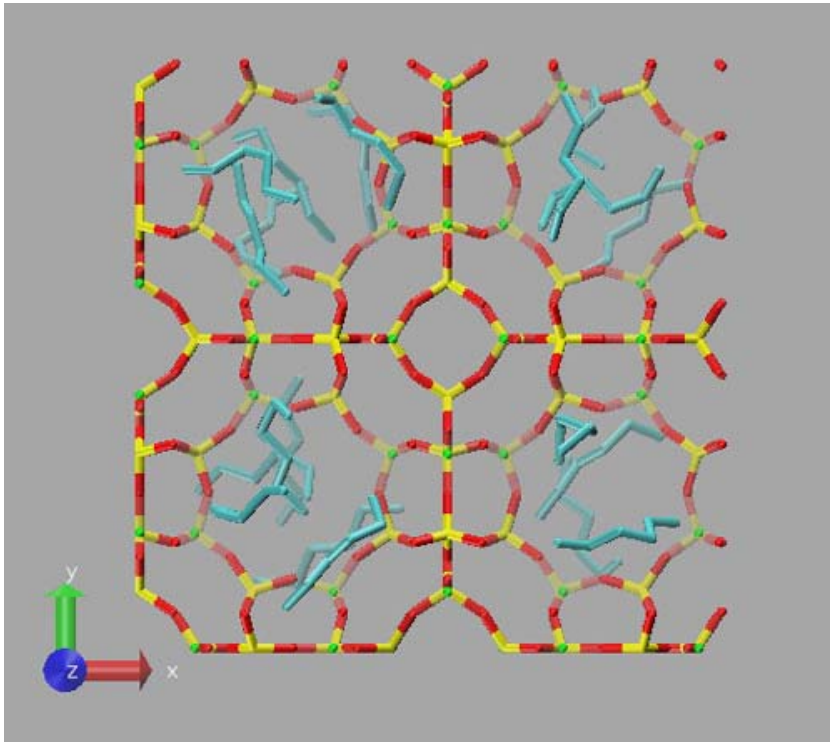

Fig. 5 Snapshot of $n$-hexane in ITQ-29-20 molecules/unit cell

\section{Conclusions}

The molecular simulations results presented in this work have a good agreement with reported experimental data. The heats of adsorption obtained by molecular simulations are in very good agreement with other published results. From this, we conclude that the UA force field is applicable to be used in predictions of adsorption equilibrium properties of linear alkanes in cation-free LTA zeolite by CBMC molecular simulation.

\section{Nomenclature}

$A_{i} \quad$ Integral intensity of the given IR band

$A_{\max }$ Integral intensity of the same band at maximum loading

$\Delta H \quad$ Heat of adsorption

$K_{S} \quad$ Sorption constant

$k_{1,2} \quad$ Constants related to the bonded interactions: bond stretching and bond bending, respectively

$q_{i} \quad$ Adsorbed phase concentration

$q_{\max }$ Maximum adsorbed phase concentration

$r \quad$ Bond length $(\AA)$

$r_{\text {cut }} \quad$ Cut-off radius

$P \quad$ Pressure

$T \quad$ Absolute temperature

V Volume

Greek letters

$\varepsilon \quad$ Characteristic energy in pair potential

$\phi$ Torsion angle

$\eta$ Constants related to torsional configurations

$\mu$ Chemical potential

$\theta \quad$ Bending angle (rad)

$\sigma \quad$ Characteristic distance in pair potential

Acknowledgements MAG thanks the financial support from Fundação para a Ciência e a Tecnologia (FCT) under the grant SFRH-BD16449/2004. TJHV acknowledges financial support from the Netherlands Organization for Scientific Research (NWO-CW) through a VIDI grant. 


\section{References}

Bates, S.P., van Well, W.J.M., van Santen, R.A., Smit, B.: Energetics of n-alkanes in zeolites: a configurational-bias Monte Carlo investigation into pore size dependence. J. Am. Chem. Soc. 118, 6753-6759 (1996)

Breck, D.W.: Zeolite Molecular Sieves. Krieger Publishing Co., Malabar (1984)

Calero, S., Dubbeldam, D., Krishna, R., Smit, B., Vlugt, T.J.H., Denayer, J.F.M., Martens, J.A., Maesen, T.L.M.: Understanding the role of sodium during adsorption: a force field for alkanes in sodium-exchanged faujasites. J. Am. Chem. Soc. 126, 1137711386 (2004)

Corma, A.: State of the art and future challenges of zeolites as catalysts. J. Catal. 216, 298-312 (2003)

Corma, A., Rey, F., Rius, J., Sabater, M.J., Valencia, S.: Supramolecular self-assembled molecules as organic directing agent for synthesis of zeolites. Nature 431, 287-290 (2004)

Dubbeldam, D.: Computer-simulation of adsorption and diffusion of hydrocarbons in zeolites. Ph.D. Thesis, University of Amsterdam (2005)

Dubbeldam, D., Beerdsen, E., Vlugt, T.J.H., Smit, B.: Molecular simulation of loading-dependent diffusion in nanoporous materials using extended dynamically corrected transition state theory. J. Chem. Phys. 122, 224712 (2005)

Frenkel, D., Smit, B.: Understanding Molecular Simulations: From Algorithms to Applications, 2nd edn. Academic Press, San Diego (2002)
Gribov, E.N., Sastre, G., Corma, A.: Influence of pore dimension and sorption configuration on the heat of sorption of hexane on monodimensional siliceous zeolites. J. Phys. Chem. B 109, 23794-23803 (2005)

Hedin, N., DeMartin, G.J., Strohmaier, K.G., Reyes, S.C.: PFG NMR self-diffusion of propylene in ITQ-29, CaA and $\mathrm{NaCaA}$ : window size and cation effects. Micropor. Mesopor. Mater. 98, 182-188 (2007)

Karavias, F., Myers, A.L.: Isosteric heats of multicomponent adsorption thermodynamics and computer simulations. Langmuir 7, 3118-3126 (1991)

Martin, M.G., Siepmann, J.I.: Transferable potentials for phase equilibria. 1. United-atom description of n-alkanes. J. Phys. Chem. B 102, 2569-2577 (1998)

Schüring, A., Auerbach, S.M., Fritzsche, S., Haberlandt, R.: On entropic barriers for diffusion in zeolites: a molecular dynamics study. J. Chem. Phys. 116, 10890 (2002)

The Atlas of Zeolite Structure Types: http://www.iza-structure.org/ databases/. Accessed November 30, 2007

Vlugt, T.J.H.: Influence of framework flexibility on the adsorption properties of hydrocarbons in the zeolite silicalite. J. Phys. Chem. B 106, 12757-12763 (2002)

Vlugt, T.J.H., Krishna, R., Smit, B.: Molecular simulations of adsorption isotherms for linear and branched alkanes and their mixtures in silicalite. J. Phys. Chem. B 103, 1102-1118 (1999) 